\title{
Biocombustibles, Seguridad Alimentaria y Cultivos Transgénicos
}

\author{
Biofuels, food security and transgenic crops
}

\author{
Orlando Acosta ${ }^{1}$ y Alejandro Chaparro-Giraldo ${ }^{2}$
}

1 Departamento de Ciencias Fisiológicas, Facultad de Medicina-Instituto de Biotecnología, Universidad Nacional de Colombia, Bogotá. oacostal@unal.edu.co

2 Departamento de Biología, Instituto de Genética. Facultad de Ciencias, Universidad Nacional de Colombia. Bogotá. achaparrog@unal.edu.co

Recibido 11 Noviembre 2008/Enviado para Modificación 20 Enero 2009/Aceptado 9 Febrero 2009

\section{RESUMEN}

El alza mundial de precios de los alimentos está amenazando con precipitar más pobres bajo la línea de pobreza, esto probablemente se agravará por el desafío que la creciente población y el cambio climático están presentando a la seguridad alimentaria. Existe evidencia de que las actividades humanas que consumen combustibles fósiles y usan tierras están contribuyendo a las emisiones de gases de invernadero y al cambio climático global. La naturaleza agotable de las reservas de combustibles fósiles y el cambio climático están suscitando preocupaciones sobre la seguridad energética, generando interés en la utilización de energías renovables como los biocombustibles. Existen preocupaciones por la producción de biocombustibles a partir de cultivos alimenticios por la posible competencia con su utilización para alimento humano y animal. Pero los biocombustibles pueden ser producidos de otras materias primas como ligno-celulosa de pastos perennes, forestales y deshechos vegetales. El contenido energético de los biocombustibles no debe exceder la energía de los combustibles fósiles utilizados en su producción, para asegurar su sostenibilidad energética, competitividad económica y aceptación ambiental. El cambio climático y los biocombustibles están desafiando los esfuerzos de la FAO para erradicar la hambruna del mundo en la próxima década. Los cultivos utilizados actualmente en la producción de biocombustibles no han sido domesticados para este fin; la tecnología transgénica de plantas puede ofrecer una enorme contribución al mejoramiento económico y ambiental de los cultivos para biocombustibles. En el presente artículo se presentan críticamente algunas de las relaciones entre biocombustibles, seguridad alimentaria y tecnología transgénica de plantas.

Palabras Clave: Biocombustibles, cambio climático, plantas modificadas genéticamente, seguridad alimentaria (fuente: DeCS, BIREME).

\section{ABSTRACT}

Soaring global food prices are threatening to push more poor people back below the poverty line; this will probably become aggravated by the serious challenge that increasing population and climate changes are posing for food security. There is growing evidence 
that human activities involving fossil fuel consumption and land use are contributing to greenhouse gas emissions and consequently changing the climate worldwide. The finite nature of fossil fuel reserves is causing concern about energy security and there is a growing interest in the use of renewable energy sources such as biofuels. There is growing concern regarding the fact that biofuels are currently produced from food crops, thereby leading to an undesirable competition for their use as food and feed. Nevertheless, biofuels can be produced from other feedstocks such as lingo-cellulose from perennial grasses, forestry and vegetable waste. Biofuel energy content should not be exceeded by that of the fossil fuel invested in its production to ensure that it is energetically sustainable; however, biofuels must also be economically competitive and environmentally acceptable. Climate change and biofuels are challenging FAO efforts aimed at eradicating hunger worldwide by the next decade. Given that current crops used in biofuel production have not been domesticated for this purpose, transgenic technology can offer an enormous contribution towards improving biofuel crops' environmental and economic performance. The present paper critically presents some relevant relationships between biofuels, food security and transgenic plant technology.

Key Words: Biofuels, climate change, food security, genetically modified plants (source: $\mathrm{MeSH}, \mathrm{NLM}$ ).

$\mathrm{E}$ 1 contexto mundial de la seguridad alimentaria cambió drásticamente en los dos últimos años. Dados sus altos precios, los alimentos se están haciendo aun más inalcanzables para los más pobres, comprometiendo seriamente su seguridad alimentaria. Como factores incidentes en esta alza de precios se señalan los altos precios internacionales del petróleo, el cambio climático y los biocombustibles $(1,2)$. A estos factores se agrega el acelerado incremento del ingreso de algunos países como China e India, lo que ha conducido a una elevada demanda por proteína de origen animal, la cual es metabólicamente construida con la ingestión de productos agrícolas (maíz, soya, pastos) utilizados en la nutrición animal, varios de ellos comunes a la nutrición humana. Un número creciente de países desarrollados y en rápido desarrollo han visto en los biocombustibles una oportunidad para depender menos de combustibles fósiles, beneficiar el ambiente y desarrollar la economía rural (3). Se estima que la actual población humana cercana a 6.5 miles de millones $(\mathrm{mml})$ de habitantes se aproximaría a 9-10 mml en el año 2050 (4), duplicándose así la demanda por alimento proveniente de la agricultura (5). La preocupación sobre el derecho a los alimentos se intensifica frente al tema de los biocombustibles, especialmente cuando estos son descritos como la conversión de alimento en combustible (6). En el presente artículo, se presenta críticamente la relación entre biocombustibles, seguridad alimentaria y la oportunidad ofrecida por la tecnología transgénica. 


\section{Métodos}

Se realizo una búsqueda sistemática de literatura publicada entre 1998 y 2008 sobre el tema de biocombustibles en sus componentes energéticos, ambientales y de seguridad alimentaria, y sobre la potencialidad de utilización de la tecnología transgénica para el mejoramiento de cultivos fuente de biocombustibles. Se accedió a las bases de datos Science Direct, Jstor y Medline, utilizando las palabras de búsqueda: "biofuels energy", "biofuels environment", " biofuels food security", "biofuels transgenic crops" y "climate change food security". Se acudió sobre los mismos temas al banco de publicaciones de la FAO y las Naciones Unidas, particularmente al Intergovernmental Panel on Climate Change (IPCC). Las relaciones entre los temas constituyeron la unidad de análisis del presente trabajo.

\section{El Tema Energético}

Recientemente una economía basada en biocombustibles renovables (etanol, biodisel, metano, hidrogeno) se proyecta hacia la reducción de la dependencia de combustibles fósiles, la disminución de emisiones de gases de invernadero (GHG) y el fortalecimiento de economías rurales (7). Sin embargo, se han expresado al respecto algunas preocupaciones ambientales y de seguridad alimentaria $(2,8)$. Los combustibles fósiles (carbón, gas natural y petróleo) contribuyen actualmente a cerca del $80 \%$ del total de las fuentes energéticas globales y sus reservas actuales durarían entre 41 y cerca de 700 años, dependiendo de los avances tecnológicos y de las tasas de producción y de consumo $(9,10)$. La naturaleza finita de estas reservas fósiles ha incrementado el interés por ampliar el portafolio energético con fuentes energéticas alternativas y renovables, tales como los biocombustibles. Se estima que el consumo de petróleo se incrementará en las próximas tres décadas, pasando de 85 millones de barriles por día en 2006 a 118 en 2030 (11), mientras que su producción presentaría su mayor pico entre 2010 y 2020. Esto, como balance, conduce a serias preocupaciones sobre la seguridad energética. En los últimos años se ha presenciado un alza vertiginosa en el precio del barril, el cual pasó de US \$ 25 en el 2000 a US \$ 140 en junio de 2008 (12). Aunque en octubre de 2008 descendió su precio a US \$ 61, los precios de los alimentos no experimentaron descensos significativos.

La producción global de biocombustibles líquidos se ha incrementado de 4.4 mml de litros en 1980 a $42.2 \mathrm{mml}$ en 2005 (13), siendo los mayores productores USA (16.1 mml), Brasil $(16 \mathrm{mml})$ y China $(3.8 \mathrm{mml})$. La producción de biodisel pasó de 11.4 millones de litros en 1991 a 3.8 mml en 2005 (14). Alemania, Francia, USA e Italia se encuentran en los primeros lugares de producción de biodisel 
(15). La producción viable de un biocombustible implica considerar beneficios ambientales comparados con los combustibles fósiles, competir económicamente con ellos, producirse en cantidades suficientes para satisfacer la demanda, y ante todo producir una ganancia energética neta (NEG) en comparación con la energía invertida en su producción (16).

Los análisis cuantitativos indican que tanto la producción de etanol y biodiesel en USA a partir de maíz y soya, respectivamente, tienen resultados positivos en términos de balance energético neto (NEB). Es decir, el contenido energético de los biocombustibles sobrepasa los insumos de energía representados en combustibles fósiles (16). Estos hallazgos desvirtúan aseveraciones sobre valores negativos de NEB para ambos biocombustibles. Sin embargo, el NEB para el etanol de los granos de maíz es relativamente pequeño, generándose cerca de $25 \%$ más energía que la consumida en en su producción, mientras que el biodiesel de soya posee un NEB de aproximadamente $93 \%$ (16).

Tanto la producción de maíz como de soya tienen efectos ambientales negativos asociados a la movilización de agroquímicos, especialmente nitrógeno, fósforo y plaguicidas (17). Aunque el etanol se puede mezclar con la gasolina como un oxigenato para reducir la cantidad de monóxido de carbono (CO), los compuestos volátiles orgánicos (VOC), la materia particulada con diámetro aerodinámico igual o menor de $10 \mathrm{~mm}$ (PM10) producida por la combustión y las emisiones totales de ciclo de vida de cinco de los mayores polutantes [CO, VOC, PM10, óxidos de azufre $\left(\mathrm{SO}_{x}\right)$ y óxidos de nitrógeno $\left(\mathrm{NO}_{\mathrm{x}}\right)$ ] se incrementan con la mezcla etanol de maíz-gasolina ("N85") en comparación con la gasolina (18). Por el contrario, la adición de pequeñas cantidades de biodiesel al diesel disminuye $\mathrm{CO}, \mathrm{VOC}, \mathrm{PM} 10$ y $\mathrm{NO}_{\mathrm{x}}$ después de la combustión, y además esta mezcla reduce las emisiones de ciclo de vida para tres de estos polutantes (VOC, PM10 y $\mathrm{SO}_{\mathrm{X}}$ ) comparado con el diesel (19). Se asume que los biocombustibles con NEB mayor de 1 reducen el $\mathrm{CO}_{2}$ liberado por los combustibles fósiles dado que las plantas remueven el $\mathrm{CO}_{2}$ de la atmosfera y que la combustión de los biocombustibles produce menos $\mathrm{CO}_{2}$.

Una fuente adicional para la producción de etanol la constituyen los componentes ligno-celulósicos de la biomasa de las plantas, tales como los pastos perennes ("swichtgrass"-pasto aguja, Panicum virgatum), residuos de cultivos y masa forestal, de los cuales se puede obtener etanol mediante sacarificación y fermentación (20). Estas fuentes etanólicas potencialmente podrían desplazar el $30 \%$ de nuestro actual consumo de petróleo (21), sin embargo, sus costos de producción se han juzgado relativamente altos (22). 
En estudios iniciales, el pasto aguja mostró un NEB de $343 \%$ cuando fue utilizado para producir etanol a partir de biomasa (23). Estudios más recientes utilizando producciones de biomasa simuladas, y estimando los insumos agrícolas, han indicado que este pasto podría producir un NEB de más $700 \%(24,25)$, o más de $540 \%$ de acuerdo con ensayos de campo (26), mientras que las emisiones de GHG se han estimado cerca de cero $(7,26)$ o ligeramente positivas $(24)$.

\section{Seguridad Alimentaria}

La FAO ha definido la seguridad al imentaria como una "situación que existe cuando toda la población, en todo momento, tiene acceso físico, social y económico a alimento suficiente, seguro y nutritivo que satisface sus necesidades dietéticas y preferencias alimentarias para una vida activa y saludable" (27). Aunque las cuatro dimensiones que esta definición comprende (disponibilidad, estabilidad, accesibilidad y utilización) no siempre son satisfechas, los escenarios del SRES ("Special Report on Emissions Scenarios") (28) describen un escenario donde el crecimiento del ingreso mundial permitirá a la mayor parte de la población mundial afrontar la escasez de alimento con la importación $(28,29)$. Se considera que los ingresos crecen a una tasa mayor que el precio real de los alimentos, lo cual indicaría que la participación del ingreso gastado en los alimentos disminuiría y que aún un incremento en los precios de los alimentos no crearía una mayor carga en el gasto alimentario de los pobres. Sin embargo, la actual situación demuestra que una inesperada alza en los precios de los alimentos está poniendo en riesgo la seguridad alimentaria de muchos pobres.

En momentos de libre comercio, se hace pertinente la discusión sobre la seguridad alimentaria en términos de si el alimento se encuentra disponible, o si los recursos monetarios y no monetarios a disposición de la población son suficientes para permitirle a cada uno el acceso a cantidades adecuadas de alimento (30). En esta discusión se ha argumentado que la autosuficiencia nacional no es necesaria ni suficiente para garantizar la seguridad alimentaria a nivel individual. Se cita el caso de Hong Kong y Singapur, que no son autosuficientes (agricultura inexistente) pero sus ciudadanos tienen seguridad alimentaria, mientras India es autosuficiente, pero una gran parte de su población no tiene seguridad alimentaria (30).

\section{Cambio Climático}

En los últimos años se ha reconocido la complejidad de los efectos del cambio climático sobre las diferentes dimensiones de la seguridad alimentaria y sobre la 
agricultura (30). Se han destacado los escenarios del SRES (28), donde el escenario $\mathrm{A} 2$ es particularmente importante para la agricultura y las fuentes alimentarias porque asume el más alto crecimiento poblacional y por lo tanto la más alta demanda por alimentos. De acuerdo con estos escenarios y los modelos climáticos considerados, se proyecta que la temperatura promedio de la superficie terrestre se elevará entre $1,8^{\circ} \mathrm{C}$ y $4,0^{\circ} \mathrm{C}$ en el 2100 (31). Estos cambios podrían conllevar algunos beneficios en las zonas (latitudes) templadas, ampliando el espectro de áreas potencialmente cultivables y eventualmente incrementando la producción agrícola, aunque algunas plagas podrían expandir su alcance. Sin embargo, las olas de calor y las sequías se podrían incrementar en la zona mediterránea, así como las precipitaciones, las inundaciones y posibles tormentas costeras en las zonas templadas (32). Las regiones semiáridas y áridas probablemente reducirían sus pastos y su producción pecuaria, y algunas llanuras tropicales podrían convertirse en regiones áridas (31).

El incremento de la concentración atmosférica de $\mathrm{CO} 2$ es otro cambio para la agricultura. En 2010, en el escenario SRES B1, el de más bajas emisiones, la concentración de CO2 pasará de cerca de 379 ppm hoy a cerca de 550 ppm, o a cerca de 880 ppm en el escenario SRES A1 $(28,31)$. Una alta concentración de $\mathrm{CO} 2$ puede tener efecto positivo sobre muchos cultivos, elevando la producción de biomasa, pero la magnitud de ese efecto no es clara dado que depende de varios factores del manejo agronómico y del tipo de cultivo (33). Se anticipa que la calidad de cultivos y tierras podría mejorar considerablemente en los países de alta latitud (desarrollados) y desmejorar en las bajas latitudes (países en desarro1lo) (29).

El cambio climático afectará todas las dimensiones de la seguridad alimentaria: disponibilidad (producción y comercio), acceso, estabilidad de fuentes de alimentos, y utilización de alimentos $(27,30,34)$. Esencialmente todas las evaluaciones cuantitativas muestran que el cambio climático intensificará la dependencia de los países en desarrollo en materia de importaciones y acentuará la inseguridad alimentaria. Muchos estudios cuantitativos indican que el ambiente socioeconómico sobre el cual impactarán los efectos del cambio climático es más importante que los impactos esperados de los efectos biofísicos mismos (30).

\section{Cultivos Transgénicos y Biocombustibles}

La revolución agrícola de los años 1960 se ha caracterizado por el uso excesivo de tierra, agua y energía, donde esta última ha provenido esencialmente de los 
combustibles fósiles a través de los fertilizantes, los plaguicidas, la irrigación y las operaciones mecanizadas, entre otros $(35,36)$. Esta preocupante dependencia de la seguridad alimentaria de unas reservas limitadas de combustibles fósiles ha sido ampliamente reconocida (37). A sus limitaciones energéticas fósiles se añade ahora la exigencia de responder doblemente a la seguridad alimentaria y a los requerimientos de los biocombustibles. Esta circunstancia plantea la necesidad de considerar la contribución de la moderna biotecnología en su versión transgénica (38). Se debe reconocer que las especies cultivadas como fuente de combustibles no han sido domesticadas para tal propósito. Se hace necesaria la supresión de genes específicos o la inserción de genes foráneos para imprimirles valor agronómico, ambiental y energético.

La humanidad ha utilizado durante milenios la combustión de materiales biológicos, con su consecuente polución. La producción de biocombustibles de primera generación suscita preocupaciones ambientales. La destilación del etanol o butanol utiliza energía derivada de la combustión del bagazo de caña, lo cual causa afecciones pulmonares. Aunque existe la prohibición mundial sobre el uso de metil bromuro para fumigar los suelos, debido a que es un potente GHG, continúan existiendo fuentes naturales de estos haluros de alcanos que reducen la capa de ozono, como es el caso de los cultivos de canola y otras especies de Brassica. Esta nociva contribución podría ser reducida significativamente a través de transgénesis, teniendo en cuenta que el gen que codifica para la síntesis de haluros de metilo ha sido caracterizado y su expresión suprimida en Arabidopsis (39). El biodisel se podría solidificar a bajas temperaturas, lo cual se podría resolver a través de modificación genética de la planta oleaginosa dirigida a obtener cadenas más cortas y más fluidas de acido graso (40).

Mediante tecnología transgénica ha sido posible duplicar el contenido total de azucares de la caña de azúcar (41). Sin embargo, la tecnología de segunda generación de biocombustibles a partir de biomasa ligno-celulósica para producir etanol tiene limitaciones en la digestión/fermentación de este sustrato. La presencia de lignina dificulta la digestión de la celulosa por parte de la celulasa. Actualmente se ha logrado incrementar la eficiencia de las celulasas y se han producido plantas transgénicas de maíz que expresan altas concentraciones de esta enzima (42). Se está intentando modificar cultivos tales como sorgo, maíz, pasto aguja, trigo y arroz mediante mejoramiento genético convencional para modificar el contenido de lignina y celulosa y para acumular carbohidratos más digeribles por carbohidrasas, pero la limitada variación en los genomas ha dificultado el objetivo (40). 
Actualmente se desarrolla un proyecto de modificación transgénica de pasto aguja para disminuir su contenido de lignina (43). Un incremento de la digestibilidad de la celulosa sería de gran impacto económico en la producción de biocombustibles líquidos. Esto se podría obtener mediante modificación transgénica para producir más celulosa biodegradable usando el gen CBD (que codifica el dominio de unión de la celulasa a la celulosa) o controlando la expresión del contenido de lignina mediante la estrategia de RNAi (RNA interferente), lo cual se ha ensayado en maíz, sorgo, pino y álamo (40). Una combinación de la tecnología transgénica con otras tecnologías, podría producir cerca de $2 \mathrm{mml}$ de toneladas de sustrato barato y de alta calidad para combustible a partir de paja, sin incrementar el área cultivada (40).

Una tercera tecnología para la generación de biocombustibles implica algas y cianobacterias como fuente de biodisel. Algunas de las limitaciones biológicas de esta iniciativa podrían ser abordadas con la transgénesis para favorecer su crecimiento, optimizar el uso de $\mathrm{CO}$, aumentar la penetración y conversión de la luz en sustratos energéticos, o mejorar su cosecha $(40,44)$. También se propone una tecnología de cuarta generación para producir biohidrógeno y bioelectricidad. Ha habido interés en capturar energía solar para producir hidrogeno y electricidad utilizando los mecanismos del aparato fotosintético natural $(40,45)$. Las primeras aproximaciones indican la necesidad de modificar la expresión de genes relacionados con la tasa de fotosíntesis oxigénica.

\section{Conclusiones}

Aunque la FAO recientemente reafirmó su compromiso con las conclusiones, el Plan de Acción y el objetivo de la Cumbre Alimentaria Mundial de reducir a la mitad la población mundial de malnutridos hacia el 2015 (46), este propósito se está viendo seriamente interferido por los desafíos provenientes de la bioenergía, el cambio climático, y las alzas en los precios de los alimentos, lo cual amenaza la seguridad alimentaria de los países en desarrollo y en transición. Los impactos del cambio climático tendrán sus mayores efectos adversos en los países cuyo desarrollo socio-económico sea más precario. Las reservas energéticas fósiles finitas pronostican una crisis energética mundial, la cual debe ser enfrentada con el concurso de todas las herramientas tecnológicas, siendo una de ellas la de los biocombustible. Se hacen necesarios estudios y evaluaciones sistemáticas sobre la viabilidad y sostenibilidad de la producción de biocombustibles derivados de diferentes fuentes. Es crucial afrontar la posibilidad de que la producción de biocombustibles no contribuya a reducir los altos precios de los combustibles, pero si contribuya consecuencialmente al alza de los precios de los alimentos. Se 
anticipa que los cultivos para biocombustibles solo serán costo-efectivos en el largo plazo si transgénicamente se les remueven toxinas, contaminantes ambientales, $\mathrm{y}$ si se hacen más productivos y óptimos como fuente de combustibles (40)

\section{REFERENCIAS}

1. FAO. Declaration of the High-Level Conference on World Food Security: The Challenges of Climate Change and Bioenergy. Food and Agriculture Organization, Rome. 2008 [Internet]. Disponible en: http://www.fao.org/fileadmin/user_upload/foodclimate/HLCdocs/declarationE.pdf Consultado Septiembre del 2008.

2. James WE, Jha S, Sumulong L, Son HH, Hasan R, Khan ME, et al. Food prices and Inflation in developing Asia: Is Poverty Reduction Coming to an End? 2008 [Internet]. Disponible en: http://www.adb.org/Documents/reports/food-prices-inflation/food-prices-inflation.pdf Consultado Octubre del 2008.

3. Pickett J, Anderson D., Bowles D, Bridgwater T, Jarvis P, Mortimer N, et al. Sustainable Biofuels: Prospects and Challenges. The Royal Society, London, UK. 2008 [Internet]. Disponible en: http://royalsociety.org/document.asp?id=7366 Consultado Octubre del 2008.

4. United Nations. World population prospects: The 2006 Revision Population Database. 2006 [internet]. Disponible en: http://esa.un.org/unpp/ Consultado Agosto del 2008.

5. Ruttan VW. Productivity growth in world agriculture: Sources and constraints. J Econ Persp 2002; 16 (4):161-184.

6. United Nations. Report to the United Nations General Assembly, submitted on 22 August 2007 by the Special UN Rapporteur on the right to food, Jean Zigler, No. A/62/289. 2007.

7. McLaughlin SB, De La Torre Ugarte DG, Jr, Garten CT, Lynd LR, Sanderson MA, Tolbert VR, at al. High-value renewable energy from prairie grasses. Environ Sci Technol 2002; 36:21222129.

8. Koh LP, Ghazoul J. Biofuels, biodiversity, and people: Understanding the conflicts and finding opportunities. Biol Conserv 2008; 141(10):2450-2460.

9. Goldemberg J, Johansson TB. World energy assessment. Overview 2004 Update. United Nations Development Program. New York, USA. 2004 [Internet]. Disponible en: http://www.undp.org/ pei/pdfs/WEAOverview.pdf Consultado Agosto del 2008.

10. Goldemberg, J. Ethanol for a sustainable energy future. Science 2007; 315:808-810.

11. EIA-DOE. International Energy Outlook 2007. Energy InformationAdministration (EIA)-US Department of Energy (DOE), Washington DC, USA. 2007 [Internet]. Disponible en: http:// www.eia.doe.gov/oiaf/ieo/index.html Consultado Octubre del 2008.

12. EIA-DOE. Official Energy Statistics from the US Government. Petroleum International Data. Energy Information Administration (EIA)-US Department of Energy (DOE), Washington DC, USA. 2008 [Intwernet]. Disponible en: http:/www.eia.doe.gov/emeu/international/cintents.html Consultado Noviembre del 2008.

13. FO Licht. World Ethanol \& Biofuels Report. Agra informa Ltd, Kent, UK. 2008 [Internet]. Disponible en: http://www.agra-net.com/portal/ Consultado Octubre del 2008.

14. Amrbruster WJ, Coyle WT. Pacific Food System Outlook 2006-2007: the future role of biofuels. Pacific Economic Cooperation Council, Singapore. 2006 [Internet]. Disponible en: http:// www. pecc.org/food/pfso-singapore2006/PECC_Annual_06_07.pdf Consultado Julio del 2008.

15. Koh LP. Potential habitat and biodiversity losses from intensified biodiesel feedstock production. Conserv Biol 2007; 21:1373-1375.

16. Hill J, Nelson E, Tilman D, Polasky S, Tiffany D. Environmental, economic and energetic costs and benefits of biodiesel and ethanol biofuels. Proc. Nat. Acad. Sci. USA 2006; 103 (30):1120611210. 
17. Vitousek PM., Aber JD, Howarth RW, Likens GE, Matson PA, Schindler DW, et al. 1997. Human alteration of the global nitrogen cycle: Sources and consequences. Ecol Appl 1997; 7:737-750.

18. Brinkman N, Wang M, Weber T, Darlington T. Well-to-Wheels Analysis of Advanced Fuel/Vehicle Systems: A North American Study of Energy Use, Greenhouse Gas Emissions, and Criteria Pollutant Emissions. Argonne Natl. Lab, Argonne, IL;2005.

19. Sheehan J, Camobreco V, Duffield J, Graboski M, Shapouri H. Life Cycle Inventory of Biodiesel and Petroleum Diesel for Use in an Urban Bus (Natl. Renewable Energy Lab, Golden, CO) NREL Publ. No. SR-580-24089; 1998.

20. Lynd LR, Cushman JH, Nichols RJ, Wyman CE. Fuel ethanol from cellulosic biomass. Science 1991; 251:1318-1323.

21. Perlack RD, Wright LL, Turhollow AF, Graham RL, Stokes BJ, Erbach DC. Biomass as Feedstock for a Bioenergy and Bioproducts Industry: The Technical Feasibility of a Billion Ton Annual Supply (Oak Ridge National Laboratory, Oak Ridge, TN) ORNL/TM-2005/66; 2005.

22. Fulton L, Howes T, Hardy J. Biofuels for transport: an international perspective, International Energy Agency (IEA), Paris, France. 2004 [Internet]. Disponible en: http://www.iea.org/ textbase/nppdf/free/2004/biofuels2004.pdf Consultado Junio del 2008.

23. McLaughlin SB, Walsh ME. Evaluating environmental consequences of producing herbaceous crops for bioenergy. Biomass Bioenergy 1998; 14:317-324.

24. Farrell $A E$, Plevin RJ, Turner BT, Jones AD, O'Hare M, Kammen DM. Ethanol can contribute to energy and environmental goals. Science 2006; 311:506-508.

25. Renewable and Applicable Energy Laboratory. Energy and Resources Group Biofuel Analysis Meta-Model. Univ of California, Berkeley, CA; 2007.

26. Schmer MR, Vogel KP, Mitchell RB, Perrin RK. Net energy of cellulosic ethanol from switchgrass. Proc Nat Acad Sci USA 2008; 105 (2):464-469.

27. FAO. The state of food insecurity in the world 2001. Food and Agriculture Organization, Rome; 2002.

28. IPCC. Special Report on Emissions Scenarios, Summary for Policy Makers, Working Group III, International Panel on Climate Change. Cambridge Univ Press, Cambridge, UK; 2000.

29. Fischer G, Shah M, van Velthuizen $\mathrm{H}$. Climate Change and Agricultural Vulnerability, A Special Report Prepared as a Contribution to the World Summit on Sustainable Development. International Institute for Applied Systems Analysis, Laxenburg, Austria; 2002.

30. Schmidhuber J, Tubiello FN. Gobal food security under climate change. Proc Nat Acad Sci USA 2007; 104 (50):19703-19705.

31. IPCC. Climate Change 2007: The Physical Science Basis, Contribution of Working Group I to the Fourth Assessment Report of the Intergovernmental Panel on Climate Change. Cambridge Univ Press, Cambridge, UK; 2007.

32. Rosenzweig C, Tubiello FN, Goldberg RA, Mills E, Bloomfield J. Increased crop damage in the U.S. from excess precipitation under climate change. Global Environ Change 2002; 12:197202.

33. IPCC. Climate Change: Impacts, Adaptation and Vulnerability, Contribution of Working Group II to the Third Assessment Report of the Intergovernmental Panel on Climate Change. Cambridge Univ Press, Cambridge, UK; 2001.

34. FAO. The State of Food Insecurity in the World 2006. Food and Agriculture Organization, Rome; 2006.

35. Giampietro M, Pimentel D. The Tightening Conflict: Population, Energy Use, and the Ecology of Agriculture. 1994 [Internet]. Disponible en: http://www.dieoff.com/page69.htm Consultado Julio del 2008.

36. Pimentel D, and Giampietro M. Food, Land, Population and the U.S. Economy, Carrying Capacity Network, 11/21/1994 [Internet]. Disponible en: http://www.dieoff.com/page55.htm Consultado Octubre del 2008.

37. Karkacier O, Goktolga ZG. Input-output analysis of energy use in agriculture. Energy Convers Manage. 2005; 46:1513-1521. 
38. Acosta O, Chaparro A. Geneticlly modifed foods and public health. Acta Biol Colomb 2008; 13 (3): 3-26.

39. Rhew RC, Østergaard L. Saltzman ES, Yanofsky MF. Genetic Control of Methyl Halide Production in Arabidopsis. Curr Biol 2003; 13:1809-1813.

40. Gressel J. Transgenics are imperative for biofuel crops. Plant Sci 2008; 174 (3):246-263.

41. Wu LG, Birch RG. Doubled sugar content in sugarcane plants modified to produce a sucrose isomer. Plant Biotechnol J 2007; 5 (1):109-117.

42. Hood EE, Love R. Lane J, Bray J, Clough R, Pappu K, et al. Subcellular targeting is a key condition for high-level accumulation of cellulase protein in transgenic maize seed. Plant Biotechnol J 2007; 5 (6):709-719.

43. Bouton JH. Molecular breeding of switchgrass for use as a biofuel crop. Curr Opin Genet Dev 2007; 17:553-558.

44. Sheehan J, Dunahay T, Benemann J, Roessler P. A Look Back at the U.S. Department of Energy's Aquatic Species Program-Biodiesel from Algae. Prepared for the U.S. Department of Energy's Office of Fuels Development, National Renewable Energy Laboratory, Golden, Colorado;1998.

45. Melis A, Melnicki MR. Integrated biological hydrogen production. Int J Hydrogen Energy 2006; 31:1563-1573.

46. FAO. Declaration of the high-level conference on world food security: The challenges of climate change and biofuels. Food and Agriculture Organization, Rome. 2008 [internet]. Disponible en: http://www.fao.org/fileadmin/user_upload/foodclimate/HLCdocs/ declaration-E.pdf Consultado Noviembre del 2008. 